\title{
Sulforaphene induces apoptosis and inhibits the invasion of esophageal cancer cells through MSK2/CREB/BCl-2 and cadherin pathway in vivo and in vitro
}

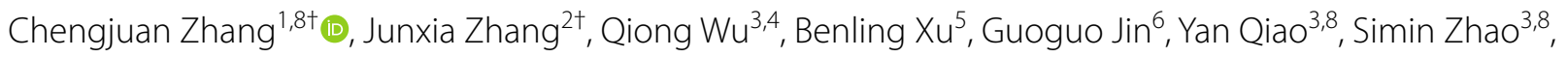
Yang Yang ${ }^{7}$, Jinwen Shang ${ }^{1}$, Xiaofang $\mathrm{Li}^{9^{*}}$ and Kangdong Liu $\mathrm{L}^{3,4,5^{*}}$

\begin{abstract}
Background: As a novel type of isothiocyanate derived from radish seeds from cruciferous vegetables, sulforaphene (SFE, 4-methylsufinyl-3-butenyl isothiocyanate) has various important biological effects, such as anti-oxidative and anti-bacterial effects. Recently, sulforaphene has attracted increasing attention for its anti-tumor effects and its ability to suppress the development of multiple tumors through different regulatory mechanisms. However, it has not yet been widely investigated for the treatment of esophageal cancer.

Methods: We observed an increased apoptosis in esophageal cancer cells on sulforaphene treatment through flow cytometry (FCM) analysis and transmission electron microscopy (TEM). Through mass spectrometry (MS) analysis, we further detected global changes in the proteomes and phosphoproteomes of esophageal cancer cells on sulforaphene treatment. The molecular mechanism of sulforaphene was verified by western blot,the effect and mechanism of SFE on esophageal cancer was further verified by patient-derived xenograft mouse model.

Results: We identified multiple cellular processes that were changed after sulforaphene treatment by proteomics. We found that sulforaphene could repress the phosphorylation of CREB through MSK2, leading to suppression of $\mathrm{BCl}-2$ and further promoted cell apoptosis. Additionally, we confirmed that sulforaphene induces tumor cell apoptosis in mice. Interestingly, we also observed the obvious inhibition of cell migration and invasion caused by sulforaphene treatment by inhibiting the expression of cadherin, indicating the complex effects of sulforaphene on the development of esophageal cancer.

Conclusions: Our data demonstrated that sulforaphene induced cell apoptosis and inhibits the invasion of esophageal cancer through a mechanism involving the inhibition of the MSK2-CREB-BCl2 and cadherin pathway. Sulforaphene could therefore serve as a promising anti-tumor drug for the treatment of esophageal cancer.
\end{abstract}

Keywords: Sulforaphene, Esophageal cancer, Apoptosis, Invasion, MSK2

*Correspondence: Ixf3@zzu.edu.cn; kangdongliu@126.com

${ }^{\dagger}$ Chengjuan Zhang and Junxia Zhang contributed equally to this work ${ }^{3}$ Department of Pathophysiology, School of Basic Medical Sciences,

Zhengzhou University, Zhengzhou, Henan, People's Republic of China

${ }^{9}$ Shangqiu Medical College, Shangqiu, Henan, People's Republic of China

Full list of author information is available at the end of the article

\section{Background}

Esophageal cancer (EC) is one of the most aggressive and common malignancies of the digestive system worldwide and has the 7th highest morbidity rate and 6th highest mortality rate $[1,2]$. There are approximately 240,000 new cases of EC in China every year, and the 5 -year overall survival rate for EC patients is still less than $25 \%[3$,

(c) The Author(s) 2019. This article is licensed under a Creative Commons Attribution 4.0 International License, which permits use, sharing, adaptation, distribution and reproduction in any medium or format, as long as you give appropriate credit to the original author(s) and the source, provide a link to the Creative Commons licence, and indicate if changes were made. The images or other third party material in this article are included in the article's Creative Commons licence, unless indicated otherwise in a credit line to the material. If material is not included in the article's Creative Commons licence and your intended use is not permitted by statutory regulation or exceeds the permitted use, you will need to obtain permission directly from the copyright holder. To view a copy of this licence, visit http://creativeco mmons.org/licenses/by/4.0/. The Creative Commons Public Domain Dedication waiver (http://creativecommons.org/publicdomain/ zero/1.0/) applies to the data made available in this article, unless otherwise stated in a credit line to the data. 
4]. EC is categorized into two major histological types: esophageal adenocarcinoma (EAC) and esophageal squamous cell carcinoma (ESCC) [5]. ESCC is the primary histological type of EC and comprises nearly $90 \%$ of EC in China [6, 7]. Although several treatments for ESCC have been developed, due to the high invasiveness and frequent regional lymph node metastasis, the prognosis of patients with ESCC is still poor [8, 9]. Recently, targeted therapy for EC showed promise [10, 11]. Novel targeted drugs have been developed and have been shown to have some therapeutic effects [12]. To improve the survival rate of EC patients, novel and effective drugs and treatment strategies are still urgently needed.

China is rich in natural Chinese herbal medicine resources; in fact, traditional Chinese medicine is a precious resource of China. The efficacy of Chinese herbal medicine has not only withstood the scrutiny of longterm medical practice but has also been confirmed by modern scientific research. Sulforaphene (SFE, 4-methylsufinyl-3-butenyl isothiocyanate) is a novel type of isothiocyanate that is derived from radish seeds from cruciferous vegetables [13]. A number of studies have indicated that sulforaphene has multiple biological functions [14, 15]. In adipocytes, sulforaphene could suppress adipogenesis through the hedgehog signaling pathway [14]. Sulforaphene could induce the expression of heme oxygenase (HO-1) and thioredoxin reductase (TrxR) in a dose-dependent manner, thus achieving detoxification effects [16]. Additionally, sulforaphene could eliminate a variety of free radicals, such as hydrogen peroxide and nitrite, and inhibit several bacteria and viruses $[17,18]$.

Recently, sulforaphene has attracted increasing attention for its anti-cancer effects in various types of cancers, such as breast cancer, ovarian cancer, hepatocellular carcinoma, and lung cancer [19-22]. Sulforaphene inhibited the development and metastasis of multiple tumors via different regulatory mechanisms [13]. In addition, sulforaphene blocked the progression of lung cancer by targeting the PI3K-AKT pathway and inhibited triplenegative breast cancer (TNBC) through activating the tumor suppressor Egr1 [23, 24]. Sulforaphene has been reported to regulate several signaling pathways involved proliferation, invasion, and apoptosis and has a significant anti-tumor effect, but it has not yet been widely investigated for the treatment of esophageal cancer. Although sulforaphene has significant anti-tumor activity and clinical research value, its potential effects on the growth of esophageal cancer cells and regulatory mechanisms remain unclear.

In this study, we revealed that sulforaphene has the potential to induce the apoptosis and inhibit invision of esophageal cancer cells in vitro and in vivo. Through proteome and phosphoproteome analyses, we identified multiple cellular processes that were changed after sulforaphene treatment. We found that sulforaphene treatment impaired the migration and invasion of cancer cells. Furthermore, sulforaphene could inhibit the expression of MSK2-CREB-Bcl-2 pathway. Meanwhile, we found that sulforaphene promoted tumor cell apoptosis in esophageal cancer in mice. In conclusion, our study revealed that sulforaphene might be a potential therapeutic agent for esophageal cancer.

\section{Materials and methods Antibodies and agents}

The following antibodies were used: tubulin (1:1000 dilution, \#ab8227, Abcam), MSK2 (1:2000 dilution, \#ab99411, Abcam), pCREBs133 (1:1000 dilution, \#9198S, CST), and Bcl2 (1:1000 dilution, \#15071, CST), Cadherin (1:500 dilution, \#ab51034, Abcam).

Sulforaphene was obtained from the Hangzhou Linan Tianhong Technology Co., Ltd., Hangzhou, Zhejiang, China and diluted in ultra-pure water. Sulforaphene was used at concentrations of $0,5,10$ and $25 \mu \mathrm{M}$ in vitro, and a dosage of 10 (low dosage) or $50 \mathrm{mg} / \mathrm{kg}$ body weight (high dosage) was used in the animal assays.

\section{Cell apoptosis assay}

Approximately $1 \times 10^{6}$ Eca109 cells were treated with DMSO or sulforaphene $(1,2.5,5,10$, or $25 \mu \mathrm{M})$ for $48 \mathrm{~h}$ and were then collected, centrifuged, and washed with PBS. Subsequently, the cells were resuspended in $100 \mu \mathrm{L}$ of binding buffer with $5 \mu \mathrm{L}$ of annexin V-FITC and incubated at room temperature for $10 \mathrm{~min}$. Subsequently, $5 \mu \mathrm{L}$ of PI solution was added, and the cells were incubated for another $5 \mathrm{~min}$ at room temperature. The apoptotic cells were detected and analyzed with a flow cytometer (Beckman Coulter, Brea, CA).

\section{Immunohistochemical assay and analysis}

To determine the expression levels of the indicated proteins in this study, immunohistochemical (IHC) assays were performed. Briefly, sample sections were fixed with $4 \%$ PFA for $30 \mathrm{~min}$ and subsequently blocked with $2 \%$ BSA for $20 \mathrm{~min}$. The slides were incubated with the indicated antibodies at room temperature for $2 \mathrm{~h}$. Subsequently, the sections were incubated with biotinylated secondary antibody for $1.5 \mathrm{~h}$, and diaminobenzidine was used as a chromogen substrate.

\section{Cell culture and transfection}

EC1 and Eca109 human esophageal cancer cells were purchased from the American Type Culture Collection (ATCC, Manassas, VA). The cells were cultured in DMEM or RPMI-1640 culture medium supplemented 
with $10 \%$ fetal bovine serum (FBS, Gibco, Grand Island, $\mathrm{CA}, \mathrm{USA})$ at $37{ }^{\circ} \mathrm{C}$ in an incubator with $5 \% \mathrm{CO}_{2}$.

\section{Phosphopeptide enrichment and LC/MS-MS measurements}

For phosphopeptide enrichment, fractionated peptide mixtures were incubated with an IMAC microsphere suspension by vibration. The IMAC microspheres with enriched phosphopeptides were collected through centrifugation, and the supernatant was subsequently removed. Mass spectrometric detection was performed on an Agilent 1290 LC system (Agilent Technologies) to an Orbitrap Q Exactive Plus mass spectrometer (Thermo Scientific). Peptide segments were dissolved in mobile phase A of liquid chromatography $(0.1 \%$ (v/v) formic acid aqueous solution) and separated by EASY-nLC 1000 ultra-high performance liquid phase system. The mobile phase $\mathrm{A}$ is an aqueous solution containing $0.1 \%$ formic acid and $2 \%$ acetonitrile, and the mobile phase $\mathrm{B}$ is an aqueous solution containing $0.1 \%$ formic acid and $90 \%$ acetonitrile. The liquid phase gradient was set at 0-48 min, 2-25\% B, 48-62 min, 25-40\% B, 62-66 $\mathrm{min}, 40-80 \% \mathrm{~B}, 66-70 \mathrm{~min}, 80 \% \mathrm{~B}$, and the flow rate was maintained at $400 \mathrm{~nL} / \mathrm{min}$., Secondary mass spectrometry data were retrieved using Maxquant.

\section{Western blot assays}

Eca109 cells were lysed to extract the total protein. Then, the samples were analyzed by SDS-PAGE. Subsequently, polyvinylidene fluoride (PVDF) membranes were blocked with 5\% milk in TBST buffer and then incubated with primary antibodies targeting MSK2, pCREB, Bcl-2, and tubulin for $1.5 \mathrm{~h}$. Then, the PVDF membranes were incubated with HRP-conjugated secondary antibodies for $1 \mathrm{~h}$. The signals were visualized with an ECL kit. Image Pro software was used to calculate the intensity of the signals in each blot.

\section{Transmission electron microscopy}

Eca109 cells were fixed in phosphate buffer $(0.1 \mathrm{M})$ containing $2.5 \%$ glutaraldehyde and $2 \%$ formaldehyde $(1.5 \mathrm{~h})$, fixed in $1 \%$ osmium tetroxide $(2 \mathrm{~h})$, dehydrated in ethanol, and embedded in epoxy resin. Then, sections were cut, stained with uranyl acetate and lead citrate, and observed using an H-7500 transmission electron microscope (Hitachi, Tokyo, Japan).

\section{Wound closure assays}

Approximately $3 \times 10^{5}$ esophageal cancer cells Eca109 were plated in 6-well plates and treated with sulforaphene at a concentration of $0,5,10$, or $25 \mu \mathrm{M}$ or DMSO and cultured as confluent monolayers. Then, a wound was mechanically generated with a $20-\mathrm{L}$ pipette tip. The cell debris was washed away twice with PBS, and complete culture medium was added to promote wound healing. The wounds were photographed at $0 \mathrm{~h}, 8 \mathrm{~h}$ and $24 \mathrm{~h}$, and the extent of wound closure in the presence of DMSO or sulforaphene treatment was measured and calculated.

\section{Transwell assays}

Esophageal cancer cells Eca109 were treated with DMSO or sulforaphene (concentration) for $48 \mathrm{~h}$ and then trypsinized and resuspended in serum-free culture medium. For the migration assays, $1 \times 10^{5}$ cells in $150 \mu \mathrm{L}$ of culture medium were added to the upper chambers of the inserts (8.0 $\mu \mathrm{m}$ membrane pores; Corning Incorporated) and cultured for $24 \mathrm{~h}$ and allowed to migrate toward the bottom chambers, which contained medium with $20 \%$ FBS.

For the Matrigel-based Transwell assays, the upper chambers of the filters were coated with 20\% Matrigel and incubated at $37{ }^{\circ} \mathrm{C}$ for $30 \mathrm{~min}$. A total of approximately $1.5 \times 10^{5}$ cells in $150 \mu \mathrm{L}$ of culture medium were then added to the upper chambers of the inserts and were allowed to migrate toward the bottom chambers, which contained medium with $20 \%$ FBS. Forty hours later, the remaining cells in the top chamber were removed, and the cells on the underside of the chamber were fixed in $4 \%$ paraformaldehyde for $20 \mathrm{~min}$ and stained with $0.2 \%$ crystal violet for $20 \mathrm{~min}$. The quantification of migrated cells was performed by dissolving crystal violet with $10 \%$ acetic acid, and the number of cells in each sample was calculated.

\section{PDX tumor growth assays}

Esophageal cancer tissue was collected from a patient diagnosed with moderate esophageal cancer. The project, which aimed to investigate the effect and molecular mechanism of sulforaphene (in humans and animals), was approved by the life science ethics review committee of Zhengzhou University and complied with the ethical requirements of biomedical research issued by international and national regulatory bodies. Written informed consent was obtained from each patient for the current study. The PDX models were initiated by the subcutaneous implantation of esophageal cancer fragments from the patient (approximately $2 \mathrm{~mm}$ ), which were coated in Matrigel and implanted through subcutaneous flap incisions. All treatment experiments were performed in C. B-17 severe combined immunodeficient mice that were 6 weeks old at the time of PDX injection/implantation. When the tumor volume reached approximately $150 \mathrm{~mm}^{3}$, the mice were randomly assigned to groups that were treated with injections of vehicle or sulforaphene. Sulforaphene was freshly prepared before each injection. The first group (10 mice) received $100 \mu \mathrm{L}$ of vehicle only (2\% DMSO and 5\% Tween-20 in PBS) every other day for 6 consecutive weeks. The other two groups 
(10 mice per group) were given $100 \mu \mathrm{L}$ of sulforaphene (dissolved in 2\% DMSO and 5\% Tween-20 in PBS) every other day at a dosage of 10 (low dose) or 50 (high dose) $\mathrm{mg} / \mathrm{kg}$ body weight for 6 consecutive weeks. The tumor volume (length $\times$ width $\times$ depth $\times 0.52$ ) was measured, and the body weights were recorded every week. Then, tumors were weighed and fixed in $10 \%$ formalin and embedded in paraffin for histological studies.

\section{Statistical analysis}

GraphPad Prism 6.0 software (GraphPad, USA) was used for the statistical analysis. All data in this study were represented as the mean \pm standard deviation (SD). Student's t-test was used for statistical comparisons. The quantifications were based on the results of at least three independent experiments. ${ }^{*}$ Indicates $\mathrm{P}<0.05$, ${ }^{* * *} \mathrm{P}<0.01$ and ${ }^{* *} \mathrm{P}<0.001$, and $\mathrm{P}<0.05$ is considered statistically significant.

\section{Results}

Sulforaphene induces apoptosis and cell cycle arrest in esophageal cancer cells

To investigate the potential of sulforaphene to induce apoptosis in esophageal cancer cells, FCM and TEM experiments were performed. FCM analysis revealed a significant increase in the total population of apoptotic cells in the presence of sulforaphene (Fig. 1a). We also noticed that both the early apoptosis rate and late apoptosis rate of esophageal cancer cells were significantly increased by sulforaphene treatment (Fig. 1c-e).

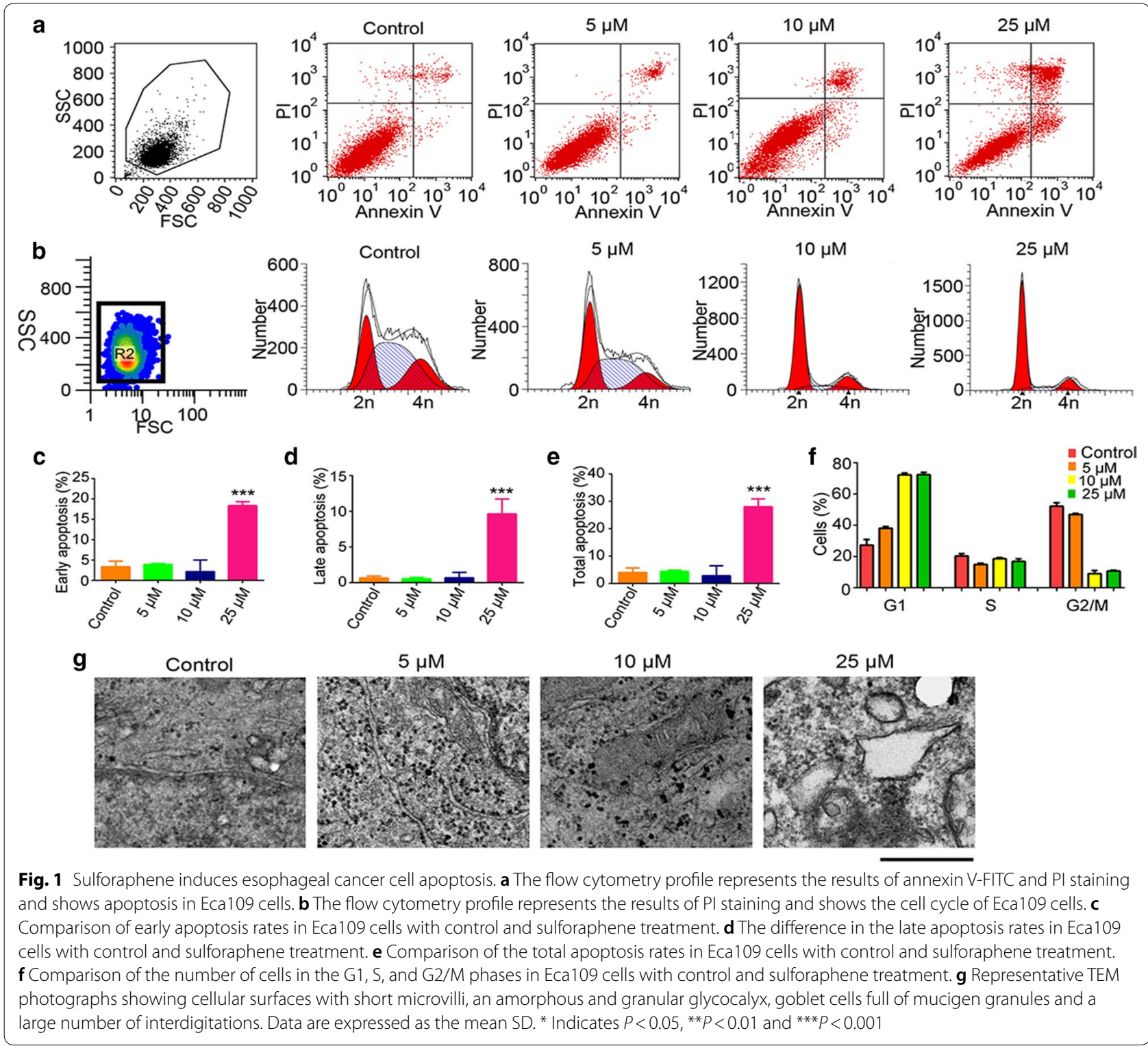


Similarly, a significant increase in cells with an apoptotic phenotype was detected through the observation of Eca109 cells treated with sulforaphene by transmission electron microscopy (TEM), as shown in Fig. 1g. As the sulforaphene concentration increased, we found that the volume of Eca109 cells decreased, the microhairs were shortened, and the chromatin became condensed. Additionally, cyto-membrane dissolution, mitochondrial swelling, and mitochondrial ridge elimination were clearly detected. Through FCM analysis, we also detected changes in the cell cycle after sulforaphene treatment. Interestingly, the results showed a significant increase in cells in the G1 phase in the presence of sulforaphene, indicating the arrest of the cell cycle (Fig. 1b, f).

\section{Sulforaphene inhibits esophageal cancer cell migration and invasion}

Subsequently, we studied the effects of sulforaphene on the migration and invasion of esophageal cancer cells. Interestingly, treatment with sulforaphene $(5,10$, or $25 \mu \mathrm{M})$ obviously inhibited the extent of wound closure in Eca109 cells (Fig. 2a-c). Furthermore, in the Transwell assays, Eca109 cells exhibited significantly decreased migration through the membranes that was caused by sulforaphene treatment $(5,10$, or $25 \mu \mathrm{M})$, and the cell numbers were obviously decreased (Fig. 2d, e). Notably, we detected the invasion capacity of Eca109 cells upon sulforaphene treatment $(5,10,25 \mu \mathrm{M})$ through Matrigelbased transwell assays and found an obvious decrease in the numbers of invading cells (Fig. 2f, g).
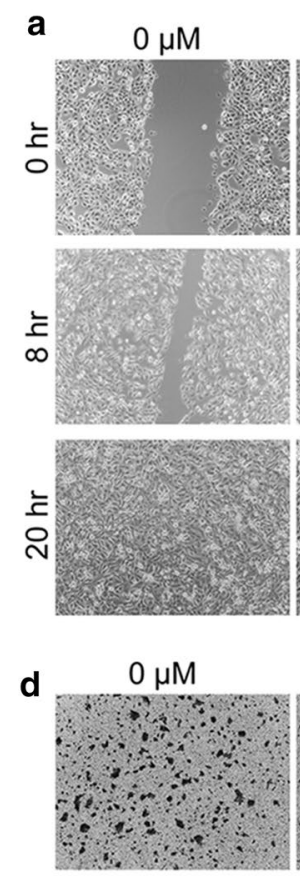

f

$0 \mu \mathrm{M}$

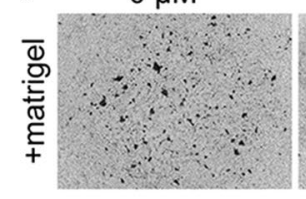

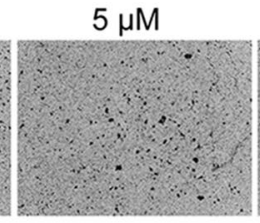

$5 \mu \mathrm{M}$
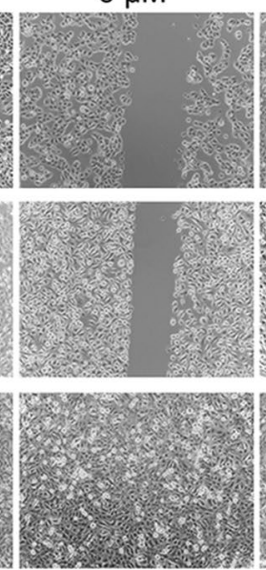

$5 \mu \mathrm{M}$

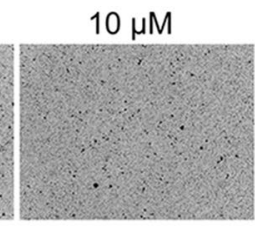

$5 \mu \mathrm{M}$

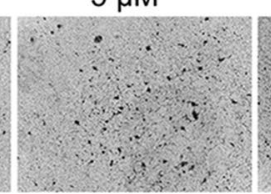

$10 \mu \mathrm{M}$
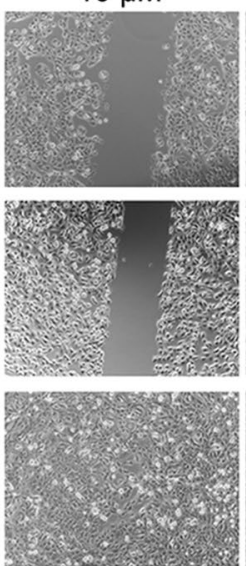

$10 \mu \mathrm{M}$

$10 \mu \mathrm{M}$

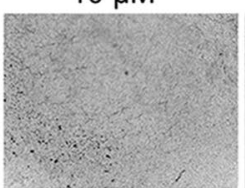

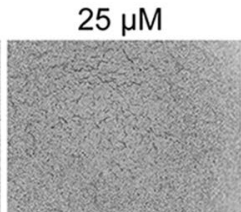

$25 \mu \mathrm{M}$
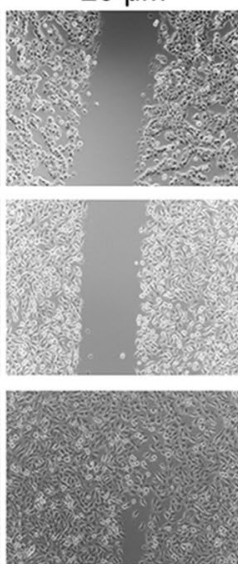

$25 \mu \mathrm{M}$

$25 \mu \mathrm{M}$

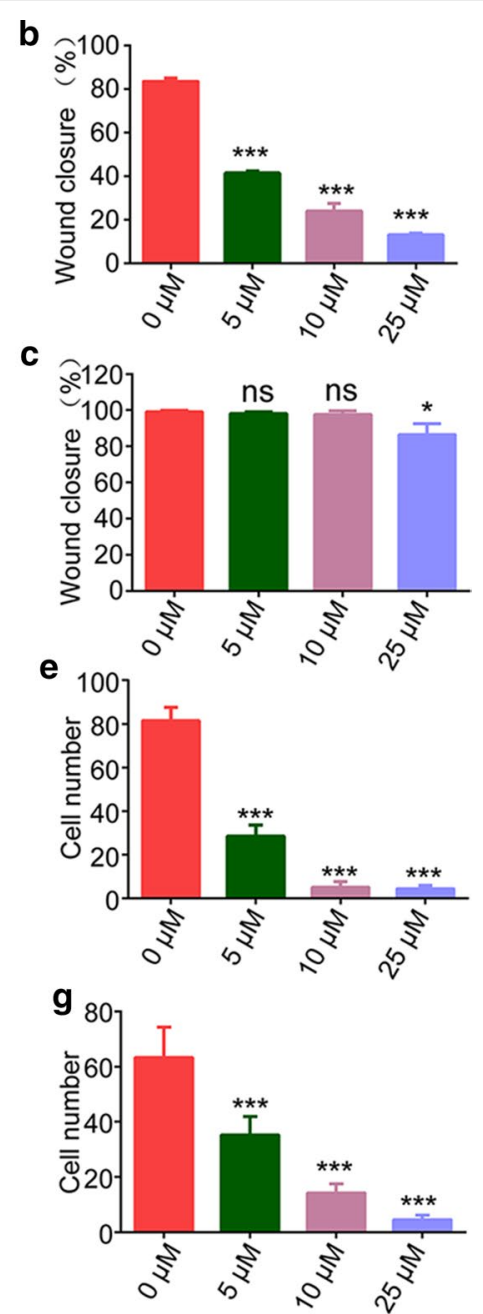

Fig. 2 Sulforaphene inhibits the migration and invasion of esophageal cancer cells in vitro. a-c Wound healing assays were performed using Eca109 cells treated with DMSO or sulforaphene $(5,10,25 \mu \mathrm{M})$, and the percentage of cell migration was measured after 8 or $20 \mathrm{~h}$. d, e Transwell assays using DMSO- or sulforaphene-treated (5, 10, or $25 \mu \mathrm{M})$ Eca109 cells were performed, and the extent of Transwell migration was quantified by counting the cells. $\mathbf{f}, \mathbf{g}$ Matrigel-based Transwell assays using DMSO- or sulforaphene-treated (5, 10, $25 \mu \mathrm{M})$ Eca109 cells were performed, and the extent of Transwell migration was quantified by cell counting. The results are presented as the mean \pm SD 


\section{Effects of sulforaphene treatment on differentially} expressed proteins and phosphorylated proteins

To gain insight into the mechanisms underlying the promotion of esophageal cancer cell apoptosis by sulforaphene, we performed mass spectrometry (MS) analysis to detect changes in the proteomes and phosphoproteomes of EC1 cells upon sulforaphene treatment, which would provide sufficient data on protein expression and phosphorylation status to identify cellular processes and signaling pathways affected by sulforaphene (Fig. 3a). For the phosphoproteome analysis, phospho-antibody mass spectrometry was used. Global protein expression and phosphorylation level changes in the presence or absence of sulforaphene were examined and analyzed. According to the results of the proteome analysis, 59 significantly upregulated proteins and 10 clearly downregulated proteins were identified for which the fold change was $>1.5$ or $<0.667$ by comparing DMSO- and sulforaphenetreated cancer cells (Fig. 3b). Similarly, we identified 320 and 97 differentially phosphorylated proteins; 248 significantly upregulated phosphorylated proteins and 79 downregulated proteins in the phosphoproteome were identified (Fig. 3c, d).

Through volcano map analysis, we found modest changes in global gene expression levels after sulforaphene treatment, and several genes were significantly upregulated and downregulated (Fig. 3e). Through localization analysis of differentially expressed genes, we found that the significantly upregulated genes were located in various parts of the cell, including the cytoplasm, nucleus and mitochondria (Fig. 3f). The significantly downregulated genes were mainly found in the cytoplasm, nucleus and endoplasmic reticulum (Fig. 3g). Additionally, a gene expression heatmap and GO analysis revealed that sulforaphene treatment remarkably affected proteins involved in the regulation of several cellular processes (Fig. 3h). Differentially expressed proteins identified from the MS analysis were further analyzed by GO analysis. Several cancer-related biological processes were found to be significantly affected by sulforaphene treatment, such as the cell cycle, cell apoptosis and cell migration (Fig. 3h).

\section{Sulforaphene could induce apoptosis through MSK2- CREB-BCl-2 pathway in esophageal cancer cells}

To further explore the molecular mechanism underlying the induction of apoptosis by sulforaphene, the western blot analysis was used and the results revealed that sulforaphene $(10 \mu \mathrm{M})$ significantly decreased MSK2, pCREB and Bcl-2 protein expression on $6 \mathrm{~h}, 12 \mathrm{~h}, 24 \mathrm{~h}$ compared with control on $0 \mathrm{~h}(P<0.05$; Fig. $4 \mathrm{a})$. We performed IHC assays to confirm the anti-apoptotic regulatory mechanism of sulforaphene in mice, the results showed the phosphorylated cadherin in tumor tissues from the sulforaphene groups than the control group (Fig. 4b). Then we valuated the effect of sulforaphene on the growth of esophageal cancer patient-derived xenografts (PDX). Tumors were isolated from mice and photographed, and the volumes of the tumors were measured every 3 days. The treatment of mice with sulforaphene reduced the mean tumor volume in the vehicle-treated group faster than that in the sulforaphene-treated (low dosage or high dosage) group (Fig. 4c). However, there were no significant differences in the body weights of mice who received control and sulforaphene treatment. Therefore, these results confirmed that sulforaphene inhibited tumor growth in mice.

\section{Discussion}

In this study, we demonstrated that sulforaphene induces apoptosis in esophageal cancer cells. We also showed that sulforaphene treatment is a potential contributor to the inhibition of the MSK2-CREB pathway and then inhibits the expression of anti-apoptotic Bcl-2. (The sequence of experimental procedures are summarized in Fig. 5). This study is an initial step in the exploration of the role of sulforaphene in esophageal cancer cell apoptosis via the MAPK signaling pathway and reveals that sulforaphene may be a promising therapeutic agent for the treatment of esophageal cancer.

Sulforaphene has been found to exhibit anticancer potential against different cancers. It was reported that sulforaphene could decreases human gastric cancer cell viability and induces apoptosis via EGFR, p-ERK1/2 down-regulation pathway [25]. Besides, sulforaphene treatment was also demonstrated to induce $\mathrm{G} 2 / \mathrm{M}$ phase cell cycle arrest and apoptosis of colon cancer cells, concomitant with phosphorylation of CDK1 and CDC25B at inhibitory sites [26]. In our study, we also verified that sulforaphene could induce apoptosis in esophageal cancer cells.

Sulforaphene plays important role in apoptosis function in various cancer cell lines but its effect on esophageal squamous cell carcinoma (ESCC) and its mechanism of action remains to be elucidated. MSK2 and its homolog MSK1, are nuclear serine-threonine kinases that belong to the ribosomal protein S6 kinase family [27]. The kinase function of MSKs activated by the upstream MAPKs extracellular signal-regulated kinase (ERK) or p38, then the activation of MSKs by ERK in response to mitogenic signals and by p38 in response to stress [28]. Additionally, the transcriptional effects of MSK2 are mediated by its phosphorylation of transcription factors, such as CREB, ATF1 (activating transcription factor 1), and NF- $\mathrm{B}$ (nuclear factor $\mathrm{\kappa B}$ ) [29]. Our results showed MSK2 and CREB activity were inhibited by sulforaphene in esophageal squamous cell carcinoma. Bcl-2 is widely known for 


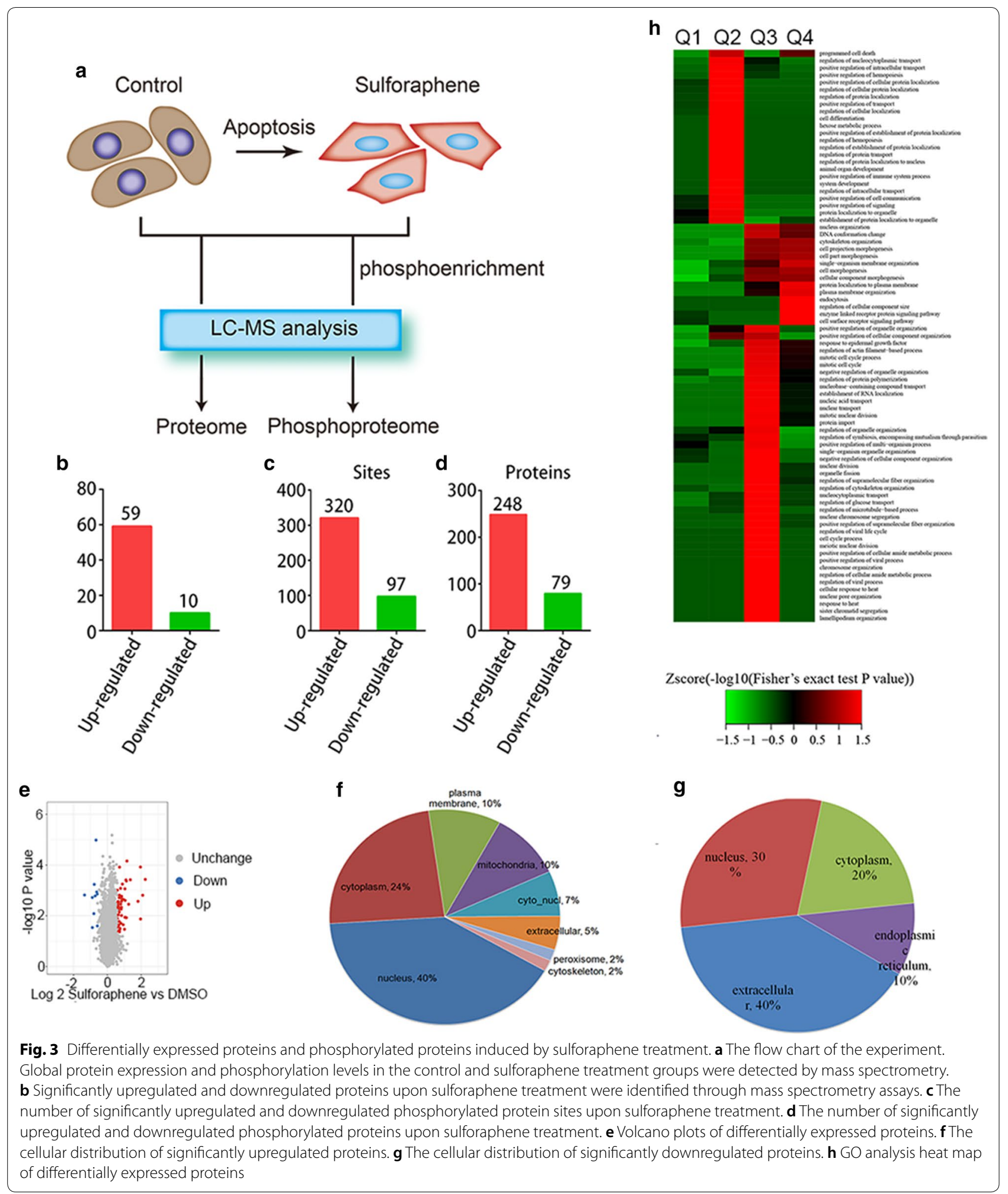

its anti-apoptotic function, and the repression of Bcl-2 expression is an available method for cancer therapy [30]. Notably, our results verified that CREB is involved in sulforaphene-induced apoptosis through down regulation of $\mathrm{Bcl}-2$ expression, which therefore mediated the induction of cell apoptosis. 


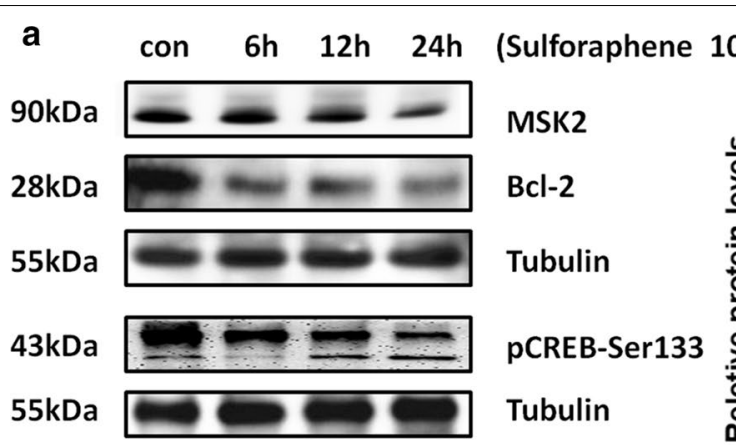

b

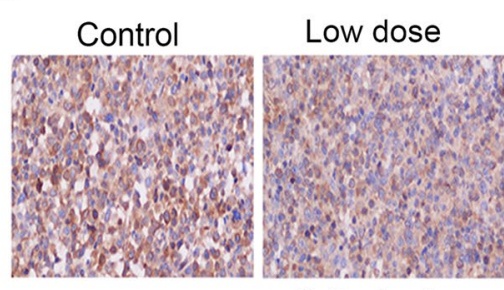

P-Cadherin

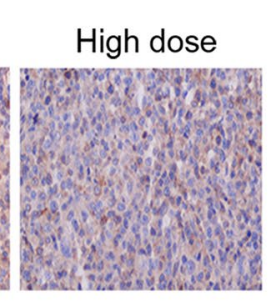

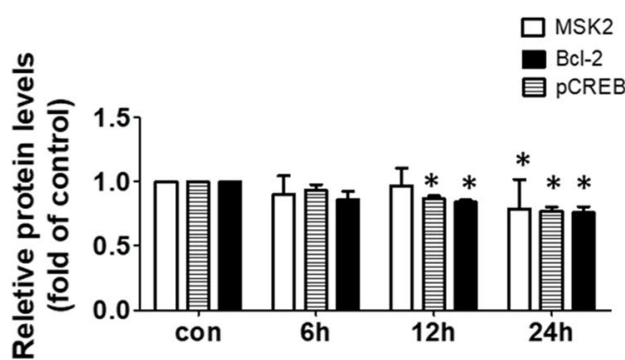

C

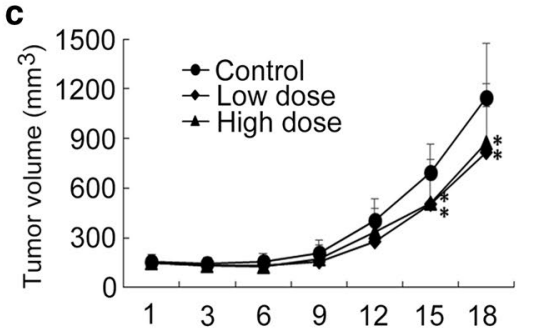

Fig. 4 Sulforaphene induce tumor apoptosis through MSK2-CREB-BCl-2 pathway. a Immunoblot analysis of the indicated MSK2, CREB and BCl-2 expression levels in Eca109 cells with quantification data $(n=3)$. $\mathbf{b} \| \mathrm{HC}$ assays showed the expression levels of $\mathrm{BCl}-2$, and $p$-cadherin in both control and sulforaphene-treated tumor tissues isolated from mice. Data are expressed as the mean \pm SD. ${ }^{*}$ Indicates $P<0.05$. $\mathbf{c}$ Comparisons of tumor volumes in representative mice in the control, low, and high sulforaphene-treated groups

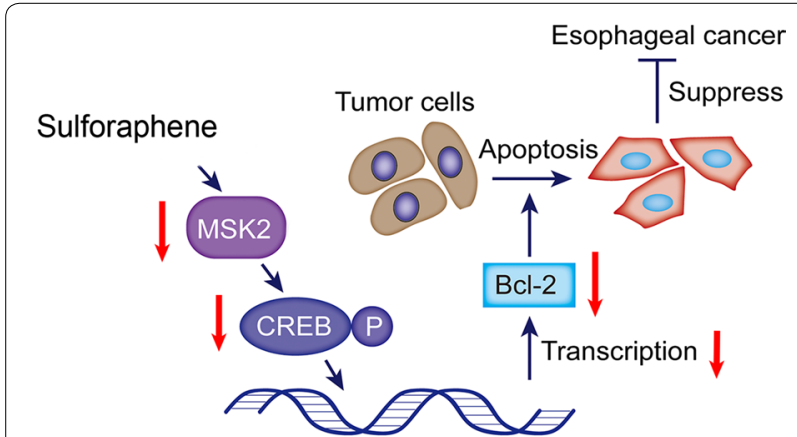

Fig. 5 Proposed molecular model of the role of sulforaphene in the esophageal cancer cell. Sulforaphene inhibited the MSK2, and CREB, to restrain the expression of the apoptosis protein $\mathrm{BCl}-2$, thereby facilitating the apoptosis of esophageal cancer cells and inhibiting cancer development

Besides the MAPK signaling pathway, other pathways and mechanisms mediate the occurrence and development of multiple types of tumors induced by sulforaphene. An increasing number of studies have demonstrated that sulforaphene promotes tumorigenesis and metastasis through the regulation of cell proliferation, migration, invasion, and apoptosis [21]. Recently, sulforaphene was reported to inhibit tumor growth in colon cancer through the induction of glutathione ablation and microtubule depolymerization [26]. Sulforaphene suppressed lung tumorigenesis by targeting the PI3K-AKT signaling pathway and enhanced the radiosensitivity of hepatocellular carcinoma via the inhibition of the NF- $\mathrm{KB}$ signaling pathway, which could also result in the apoptosis of HCC cells [21, 23]. Interestingly, sulforaphene induces mitophagic cell death via p62/SQSTM1 accumulation and AMPK inhibition [15].

Here, we found that sulforaphene could dramatically induce apoptosis and cell cycle arrest in esophageal cancer cells and effectively inhibit tumor growth in mice. Similarly, our previous study confirmed that a promising agent, HOI-02, facilitated apoptosis and cell cycle arrest in ESCC through ROS. Additionally, a previous study indicated that sulforaphene caused cytotoxicity and promoted the apoptosis of human hepatocarcinoma HepG2 cells via increases in caspase 3 and 9 activity [31]. Sulforaphene could also promote human gastric cancer cell apoptosis by downregulating EGFR and p-ERK1/2 and inhibiting the MAPK signaling pathway [25, 32]. In addition, another study demonstrated that a combination of sulforaphene and carboplatin could effectively inhibit proliferation and induce apoptosis in human NSCLC A549 cells through cell cycle arrest, caspase inhibition and mitochondrial membrane potential disruption [22]. Notably, our findings revealed the molecular mechanism underlying the stimulation of esophageal cancer cell apoptosis by sulforaphene. However, previous studies reported that apoptosis and senescence phenomena may occur when 
stress happened to cells. It also demonstrated that some cells are prone to senescence rather than apoptosis after low doses of radiation [33]. Therefore, in the next experiment, we will continue to study whether sulforaphene have the effect on senescence of esophageal cancer cells.

Tumor metastasis is induced by the invasion of tumor cells into blood vessels, and P-cadherin has been widely reported to be involved in this process. In this study, we confirmed that sulforaphene inhibited cell migration and invasion in esophageal cancer through the inhibition of P-cadherin expression. Similarly, a previous study revealed that P-cadherin inhibited the suppression of invasion by E-cadherin through the disruption of E-cadherin/p120-cadherin complexes near the cell cortex. We next need to confirm whether sulforaphene inhibits esophageal cancer cell migration through a similar regulatory mechanism.

\section{Conclusion}

Our findings, together with those of other studies, indicated that sulforaphene could repress MSK2, and CREB, Bcl-2, down-regulate the C-cadherin expression and thereby induced apoptosis and inhibited invasion in esophageal cancer cells. We would next investigated whether sulforaphene could inhibit the proliferation of esophageal cancer cells through these reported signaling pathways according to other studies with sulforaphene. To be concluded, sulforaphene could have the potential to serve as an anti-tumor drug through the promotion of tumor cell apoptosis and the inhibition of cell invasion. Which could be effectively used in the clinical treatment of esophageal cancer.

\section{Abbreviations}

ESCC: esophageal squamous cell carcinoma; FCM: flow cytometry; TEM: transmission electron microscopy; MS: mass spectrometry; DMSO: dimethylsulfoxide; CREB: CAMP-response element binding protein; PDX: patient-derived tumor xenograft.

\section{Acknowledgements}

We would like to thank PTM BioLab, Inc., for performing the proteomics and phosphorylation analyses in this study.

\section{Authors' contributions}

CJZ, JXZ, XFL and KDL conceived the study and participated in its design, QW, YQ and GGJ contributed to the acquisition of data and experimentation on animals, SMZ, BLX, and JWS performed the IHC analyses, and CJZ and YY drafted the manuscript. All authors read and approved the final manuscript.

\section{Funding}

This work was supported by The Funding Program for the National Natural Science Foundation (Project \# 81602637 and 81572812).

\section{Availability of data and materials}

The datasets supporting the conclusions of this study are included in this article. Any requests for data or materials can be sent to the corresponding author.

\section{Ethics approval and consent to participate}

The project on the effects and molecular mechanisms of sulforaphene (in humans and animals was approved by the life science ethics review committee of Zhengzhou University and complied with the ethical requirements for biomedical research issued by international and national regulatory bodies). Written informed consent from each patient was obtained for the current study.

\section{Consent for publication}

Not applicable.

\section{Competing interests}

The authors declare that they have no competing interests.

\begin{abstract}
Author details
${ }^{1}$ Biorepository Center, The Affiliated Cancer Hospital of Zhengzhou University, Henan Cancer Hospital, Zhengzhou, Henan, People's Republic of China. ${ }^{2}$ Experimental Research Center, Henan University of Chinese Medicine, Zhengzhou, Henan, People's Republic of China. ${ }^{3}$ Department of Pathophysiology, School of Basic Medical Sciences, Zhengzhou University, Zhengzhou, Henan, People's Republic of China. ${ }^{4}$ China-US (Henan) Hormel Cancer Institute, Zhengzhou, Henan, People's Republic of China. ${ }^{5}$ Department of Immunotherapy, The Affiliated Cancer Hospital of Zhengzhou University, Henan Cancer Hospital, Zhengzhou, Henan, People's Republic of China.

${ }^{6}$ Laboratory of Bone Tumor, Henan Luoyang Orthopedic Hospital, Zhengzhou, Henan, People's Republic of China. ${ }^{7}$ Clinical Systems Biology Research Center, The First Affiliated Hospital of Zhengzhou University, Zhengzhou, Henan, People's Republic of China. ${ }^{8}$ Provincial Cooperative Innovation Center for Cancer Chemoprevention, Zhengzhou University, Zhengzhou, Henan, People's Republic of China. ${ }^{9}$ Shangqiu Medical College, Shangqiu, Henan, People's Republic of China.
\end{abstract}

Received: 10 February 2019 Accepted: 9 December 2019

Published online: 19 December 2019

\section{References}

1. Mizuta $\mathrm{H}$, et al. Predictive factors for esophageal stenosis after endoscopic submucosal dissection for superficial esophageal cancer. Dis Esophagus. 2009;22(7):626-31.

2. Di Pardo BJ, et al. The global burden of esophageal cancer: a disabilityadjusted life-year approach. World J Surg. 2016;40(2):395-401.

3. Lu HB. MicroRNA-556-3p promotes the progression of esophageal cancer via targeting DAB2IP. Eur Rev Med Pharmacol Sci. 2018;22(20):6816-23.

4. Zhang SW, et al. Mortality and survival analysis of esophageal cancer in China. Zhonghua Zhong Liu Za Zhi. 2016;38(9):709-15.

5. He Y, et al. Incidence and mortality rate of esophageal cancer has decreased during past 40 years in Hebei Province, China. Chin J Cancer Res. 2015;27(6):562-71.

6. Wang $M$, et al. Distribution of esophageal squamous cell cancer and precursor lesions in high-risk areas, Linzhou in Henan province and Feicheng in Shandong province of China, 2005-2009. Zhonghua Yu Fang Yi Xue Za Zhi. 2015;49(8):677-82.

7. Sun $X$, et al. The long-term spatial-temporal trends and burden of esophageal cancer in one high-risk area: a population-registered study in Feicheng, China. PLoS ONE. 2017;12(3):e0173211.

8. Duan XF, et al. The prevalence of lymph node metastasis for pathological T1 esophageal cancer: a retrospective study of 143 cases. Surg Oncol. 2018;27(1):1-6.

9. Yang Z, et al. Upregulation of PDK1 associates with poor prognosis in esophageal squamous cell carcinoma with facilitating tumorigenicity in vitro. Med Oncol. 2014;31(12):337.

10. Zhang L, et al. Targeted therapy in esophageal cancer. Expert Rev Gastroenterol Hepatol. 2016;10(5):595-604. 
11. Gaur P, Kim MP, Dunkin BJ. Esophageal cancer: recent advances in screening, targeted therapy, and management. J Carcinog. 2014;13:11.

12. Wang F, Fan QX. Current status and future prospect of internal medicine treatment for advanced esophageal cancer. Zhonghua Zhong Liu Za Zhi. 2016;38(9):655-9.

13. Chatterjee $S$, et al. Sulforaphene enhances the efficacy of photodynamic therapy in anaplastic thyroid cancer through Ras/RAF/MEK/ERK pathway suppression. J Photochem Photobiol B. 2018;179:46-53.

14. Chen J, et al. Sulforaphene inhibition of adipogenesis via hedgehog signaling in 3T3-L1 adipocytes. J Agric Food Chem. 2018;66(45):11926-34.

15. Wang $\mathrm{H}$, et al. Traditional herbal medicine-derived sulforaphene promotes mitophagic cell death in lymphoma cells through CRM1-mediated p62/SQSTM1 accumulation and AMPK activation. Chem Biol Interact. 2018;281:11-23.

16. He C, et al. Sulforaphane attenuates homocysteine-induced endoplasmic reticulum stress through Nrf-2-driven enzymes in immortalized human hepatocytes. J Agric Food Chem. 2014;62(30):7477-85.

17. Ko MO, Kim MB, Lim SB. Relationship between chemical structure and antimicrobial activities of isothiocyanates from cruciferous vegetables against oral pathogens. J Microbiol Biotechnol. 2016;26(12):2036-42.

18. Shishu, Singla AK, Kaur IP. Inhibition of mutagenicity of food-derived heterocyclic amines by sulphoraphene-an isothiocyanate isolated from radish. Planta Med. 2003;69(2):184-6.

19. Bao C, et al. Sulforaphene interferes with human breast cancer cell migration and invasion through inhibition of hedgehog signaling. J Agric Food Chem. 2016;64(27):5515-24.

20. Biswas R, Ahn JC, Kim JS. Sulforaphene synergistically sensitizes cisplatin via enhanced mitochondrial dysfunction and PI3K/PTEN modulation in ovarian cancer cells. Anticancer Res. 2015;35(7):3901-8.

21. Ren K, et al. Sulforaphene enhances radiosensitivity of hepatocellular carcinoma through suppression of the NF-kappaB pathway. J Biochem Mol Toxicol. 2017;31(8):e21917.

22. Chatterjee S, Rhee YH, Ahn JC. Sulforaphene-carboplatin combination synergistically enhances apoptosis by disruption of mitochondrial membrane potential and cell cycle arrest in human non-small cell lung carcinoma. J Med Food. 2016;19(9):860-9.
23. Yang $M$, et al. The natural compound sulforaphene, as a novel anticancer reagent, targeting PI3K-AKT signaling pathway in lung cancer. Oncotarget. 2016;7(47):76656-66.

24. Yang M, et al. Sulforaphene inhibits triple negative breast cancer through activating tumor suppressor Egr1. Breast Cancer Res Treat. 2016;158(2):277-86.

25. Mondal A, et al. Sulforaphene promotes Bax/BCl2, MAPK-dependent human gastric cancer AGS cells apoptosis and inhibits migration via EGFR, p-ERK1/2 down-regulation. Gen Physiol Biophys. 2016;35(1):25-34.

26. Byun $\mathrm{S}$, et al. Sulforaphene suppresses growth of colon cancer-derived tumors via induction of glutathione depletion and microtubule depolymerization. Mol Nutr Food Res. 2016;60(5):1068-78.

27. Deak M, et al. Mitogen- and stress-activated protein kinase-1 (MSK1) is directly activated by MAPK and SAPK2/p38, and may mediate activation of CREB. EMBO J. 1998;17(15):4426-41.

28. Llanos S, Cuadrado A, Serrano M. MSK2 inhibits p53 activity in the absence of stress. Sci Signal. 2009;2(89):ra57.

29. Wiggin GR, et al. MSK1 and MSK2 are required for the mitogen- and stress-induced phosphorylation of CREB and ATF1 in fibroblasts. Mol Cell Biol. 2002;22(8):2871-81.

30. Czabotar PE, et al. Control of apoptosis by the BCL-2 protein family: implications for physiology and therapy. Nat Rev Mol Cell Biol. 2014;15(1):49-63.

31. Kntayya SB, et al. Induction of apoptosis and cytotoxicity by isothiocyanate sulforaphene in human hepatocarcinoma HepG2 cells. Nutrients. 2018;10(6):718.

32. Smith $A$, et al. Imidazo[1,2-a]pyridin-6-yl-benzamide analogs as potent RAF inhibitors. Bioorg Med Chem Lett. 2017;27(23):5221-4.

33. Alessio $\mathrm{N}$, et al. Low dose radiation induced senescence of human mesenchymal stromal cells and impaired the autophagy process. Oncotarget. 2015;6(10):8155-66

\section{Publisher's Note}

Springer Nature remains neutral with regard to jurisdictional claims in published maps and institutional affiliations.
Ready to submit your research? Choose BMC and benefit from:

- fast, convenient online submission

- thorough peer review by experienced researchers in your field

- rapid publication on acceptance

- support for research data, including large and complex data types

- gold Open Access which fosters wider collaboration and increased citations

- maximum visibility for your research: over $100 \mathrm{M}$ website views per year

At BMC, research is always in progress.

Learn more biomedcentral.com/submissions 